\title{
The Development of Selected Cardiovascular Parameters in Patients With Type 2 Diabetes Mellitus During a Spa Treatment
}

\author{
E. FIALOVÁ ${ }^{1}$, O. KITTNAR ${ }^{1}$ \\ ${ }^{1}$ Institute of Physiology, First Faculty of Medicine, Charles University in Prague, Czech Republic
}

Received March 31, 2015

Accepted July 29, 2015

On-line December 15, 2015

\section{Summary}

Diabetes mellitus is not just a simple metabolic disorder, however, it is considered to be a cardiovascular disease of a metabolic origin. This is apparent especially when speaking about type 2 diabetes (DM II). The objective of our study was to determine whether a comprehensive spa treatment (procedures and drinking cure) may affect the level of the sympathetic tone of patients suffering from DM II. As an indicator of the sympathetic tone, selected electrocardiographic parameters derived from the heart rate variability and microwave alternans were chosen. There were 96 patients enrolled in our study: 38 patients with poorly controlled DM II and two control groups: 9 patients with compensated DM II and 49 patients, average age without diabetes or other disorders of the glucose metabolism. All received an identical spa treatment and continued their medical therapy. The electrophysiological examination of patients was performed before and after a three-week spa treatment using the KARDiVAR system. Parameters derived from the analysis of heart rate variability (HRV), microvolt T-wave alternans, and microvolt R-wave alternans were analyzed in order to evaluate the tones of the autonomic nervous system (ANS). The control group showed a slight increase of parameter the index of activity of regulatory systems (IRSA) (4.4 \pm 1.3 vs. $3.8 \pm 1.4 ; p=0.006)$ after the spa treatment, while increased heart rate $(80.9 \pm 11.0$ vs. $74.6 \pm 9.6 ; p=0.028)$, reduced index of centralization (IC) $(1.3 \pm 0.6$ vs. $2.9 \pm 1.4 ; \mathrm{p}=0.027)$ and reduced index of myocardium (IM) (9.9 \pm 7.4 vs. $18.0 \pm 6.3 ; p=0.041)$ were found in patients with a compensated DM II. Patients with a poorly compensated DM II showed a decreased IM (10.9 \pm 8.6 vs. $16.9 \pm 5.2 ; \mathrm{p}=0.001)$ and also a reduced IRSA ( $4.1 \pm 3.5$ vs. $6.3 \pm 1.9 ; p=0.001)$. The results proved favorable changes in ANS cardiovascular control of patients with DM II after a spa treatment, especially in terms of reducing the sympathoadrenal system activity (decreased IRSA), improving electrical stability of
\end{abstract}

the myocardium and increasing centrally controlled heart rate variability without overloading the cardiovascular system (drop of IM).

\section{Key words}

Diabetes mellitus type II • ECG • Heart rate variability • Spa treatment

\section{Corresponding author}

O. Kittnar, Institute of Physiology, First Faculty of Medicine, Charles University in Prague, Albertov 5, 12800 Prague 2, Czech Republic. Fax: 224918 816. E-mail: otomar.kittnar@If1.cuni.cz

\section{Introduction}

Diabetes mellitus is not just a simple metabolic disorder, but it is considered to be a cardiovascular disease of a metabolic origin. This is apparent especially when speaking about type 2 diabetes (DM II) (Hennersdorf et al. 2000). Patients suffering from diabetes mellitus (DM) have high occurrence of the autonomic nervous system disorders, particularly disorders of balance between the sympathetic (SNS) and parasympathetic (PSNS) nervous system in terms of increased activity of the SNS. These disorders correlate with peripheral autonomic neuropathy and are considered to be one of the major pathophysiological mechanisms of development of DM II and also a factor that increases the level of a cardiometabolic risk (Stuckey and Petrella 2013). Diabetic autonomic neuropathy causes substantial morbidity and increases mortality, more specifically if cardiovascular autonomic neuropathy is present (Bissinger et al. 2014).

An increased tone of the central SNS leads to 
an increased heart rate and cardiac output and yet, at the same time, to an increased peripheral resistance due to vasoconstriction and an increased reabsorption of sodium ions and reabsorption of water in the kidneys. These mechanisms logically lead to an increased arterial blood pressure which involves a number of other mechanisms caused by hyperinsulinemia which is a compensation mechanism of the insulin resistance. From the clinical point of view, it is not critical which of the regulatory mechanisms is primary, i.e. whether it is the insulin resistance or SNS.

The heart is a rather sensitive indicator of all events occurring in the body. The heart rhythm, but also the rate of its time changes that is influenced by the sympathetic and parasympathetic part of the autonomic nervous system, is very sensitive to any stress influence and, additionally, it reflects the impact of higher levels of regulation. Patients with DM have an increased heart rate, extended QTc section (Palova et al. 2010), increased dispersion of the QT interval (Zdarska et al. 2007) and reduced heart rate variability (HRV) (Stuckey and Petrella 2013).

One of the options of influencing the increased SNS tone and reducing the risk of further development of DM (and a way of a successful compensation of DM) is a spa treatment (ST), as a part of a comprehensive approach to treatment and rehabilitation of these patients.

Hypothalamus plays the key role in balneology and balneotherapy. It is an integral part of the limbic system and the control center of the autonomic nervous system. Thanks to the fact that balneology can affect stress tension of an organism and metabolic processes at the same time, we can purposefully influence functions of various tissues and internal organs. This was shown in the results of a research team of physicians from Luhačovice who demonstrated that a spa treatment resulted in positive changes of the glycosylated proteins index in $88.9 \%$ of cases of patients suffering from DM (Jandova 2009). Moreover, some other studies have shown that strict glycemic control leads to improvement of the autonomic nervous system (Vinik et al. 2003, Ziegler 1994).

The objective of our study was to determine whether a comprehensive spa treatment (procedures and drinking cure) may affect the level of the sympathetic tone of patients suffering from DM II. As an indicator of the sympathetic tone, selected electrocardiographic parameters derived from the heart rate variability and microwave alternans were chosen.

\section{Material and Methods}

The study was carried out in Mariánské Lázně in 2012-2014 where several balneological sources were used for the spa treatment: water springs (drinking cures and mineral baths which contain free $\mathrm{CO}_{2}$ that is dissolved in water in a dissociated form), carbon dioxide gas springs (used as dry gas envelopes or gas injections) and bog. Apart from the above stated balneological procedures, the patients' plan included physiatric procedures, dietary measures and exercise therapy. The treatment plan also included control and elimination of risk factors.

There were 96 patients enrolled in the examination: 38 patients with poorly controlled DM II who met the criteria for inclusion in the study: the level of blood glucose 6.5-13.7 mmol/1, levels of glycosylated hemoglobin higher than $6.0 \%$ and average age of $62.7 \pm$ 8.0 years. Two control groups were included in the study: 9 patients with compensated DM II with a maximum level of blood glucose $6.5 \mathrm{mmol} / 1$, glycosylated hemoglobin level up to $4.0 \%$ and an average age of $62.7 \pm 9.2$ years, and a group of 49 patients, average age $60.4 \pm 7.4$ years who were without diabetes or other disorders of the glucose metabolism. Patients suffering from any confirmed cardiovascular disease were excluded from the study. All patients included in the study received an identical spa treatment and continued their medical therapy. In all patients basic biochemical examinations were performed (including plasma levels of glycaemia, lipid spectrum, creatinine, urea etc.).

The electrophysiological examination of patients was performed before and after a three-week spa treatment using the KARDiVAR system. The system helps perform both records of electrocardiogram signal from 3 standard limb leads and process and analyze the signal. The method is used to examine the current state of the autonomic nervous system and carry out an analysis of risk factors and adaptive capabilities of the organism. The measurements were carried out under standard conditions in a relative calm, first 1.5-2 $\mathrm{h}$ after eating. The ECG signal was recorded in a sitting position and the patient was performing a regular and quiet breathing. The duration of the autonomous recording of the ECG signal was 5 min.

The following parameters derived from the analysis of HRV, microvolt T-wave alternans, and microvolt R-wave alternans were evaluated:

- The heart rate (HR) (Xhyheri et al. 2012). HR is the simplest indicator of the current setting of the autonomic nervous system. 
- The index of centralization (IC) (Baevsky et al. 1997). IC indicates the degree of non-respiratory sinus arrhythmia prevalence in comparison with the respiratory ones, and is a quantitative characteristic of the ratio between the central and autonomic level of control of the heart rhythm. It is calculated from the data of the spectral analysis of the HRV: slow waves of the first (LF) and second order (VLF), characterizing the sympathetic activity of the vasomotor waves, the state of the regulatory system of the vascular tone and the high-frequency waves (HF), characterizing the ratio between vagal and sympathetic modulation of the heart rhythm.

- Myocardium index (IM) (Ivanov et al. 1996). IM is a summation parameter of the analysis of microwaves alternans which shows the integral degree of a deviation from normal dispersion curve values (the areas beyond standards). Dispersion characteristics are calculated from intervals of a complete ECG PQRST interval and are divided into 9 groups of deviations (G1-G9): G1-2 - dispersion deviations during depolarization of the atria, G3-4 - deviations during depolarization of ventricles, G5-6 - deviations of repolarization of ventricles, G7 - depolarization symmetry in the middle parts of the QRS complex, G8 - ventricular conduction disorder, G9 - depolarization symmetry in the initial part of the QRS complex. The IM reaches values of $0-100 \%, I M=0-$ total absence of changes, IM $<15 \%$ - small deviations from the standard, $15-21 \%$ are threshold values.

- Index of activity of regulatory systems (IRSA) (Baevskii et al. 1983). IRSA evaluates HRV comprehensively and allows: distinguishing of different degrees of regulatory system stresses and evaluation of the adaptability of an organism. The computational algorithm includes parameters of statistical histogram and spectral analysis of the HRV. The values are then expressed on a scale from 1 to 10 which represents the states from the optimal (physiological predominance of the parasympathetic tone) to the state of depletion of the regulatory mechanisms (with the maximum sympathetic tone and parasympathetic influence suppressed).

The data analysis was performed using the following statistical methods: Shapiro-Wilk normality test, Wilcoxon non-parametric test for two dependent selections, Kruskal-Wallis non-parametric test for 2 independent selections, test of dependency $\chi^{2}$ (chi-square).

Table 1. Studied parameters in all three groups before and after ST.

\begin{tabular}{|c|c|c|c|c|c|c|}
\hline & \multicolumn{2}{|c|}{ Control group } & \multicolumn{2}{|c|}{ Well compensated DM } & \multicolumn{2}{|c|}{ Poorly compensated DM } \\
\hline & Before spa & After spa & Before spa & After spa & Before spa & After spa \\
\hline \multirow{2}{*}{$H R$} & $68.3 \pm 7.9$ & $68.4 \pm 12.3$ & $74.6 \pm 9.6$ & $80.9 \pm 11.0$ & $78.3 \pm 12.9$ & $77.9 \pm 11.5$ \\
\hline & \multicolumn{2}{|c|}{$\mathrm{p}=0.129$} & \multicolumn{2}{|c|}{$\mathrm{p}=\mathbf{0 . 0 2 8}$} & \multicolumn{2}{|c|}{$\mathrm{p}=0.989$} \\
\hline \multirow{2}{*}{$I C$} & $2.4 \pm 2.1$ & $2.4 \pm 1.9$ & $2.9 \pm 1.4$ & $1.2 \pm 0.6$ & $3.3 \pm 2.9$ & $3.5 \pm 3.1$ \\
\hline & \multicolumn{2}{|c|}{$\mathrm{p}=0.546$} & \multicolumn{2}{|c|}{$\mathbf{p}=\mathbf{0 . 0 2 7}$} & \multicolumn{2}{|c|}{$\mathrm{p}=0.798$} \\
\hline \multirow{2}{*}{$I M$} & $14.8 \pm 3.1$ & $15.7 \pm 4.7$ & $18.0 \pm 6.3$ & $9.9 \pm 7.4$ & $16.9 \pm 5.2$ & $10.9 \pm 8.6$ \\
\hline & \multicolumn{2}{|c|}{$\mathrm{p}=0.489$} & \multicolumn{2}{|c|}{$p=0.041$} & \multicolumn{2}{|c|}{$\mathrm{p}=\mathbf{0 . 0 0 1}$} \\
\hline \multirow{2}{*}{ IRSA } & $3.8 \pm 1.4$ & $4.4 \pm 1.3$ & $5.4 \pm 2.8$ & $4.2 \pm 3.5$ & $6.3 \pm 1.9$ & $4.1 \pm 3.5$ \\
\hline & \multicolumn{2}{|c|}{$p=0.006$} & \multicolumn{2}{|c|}{$\mathrm{p}=0.351$} & \multicolumn{2}{|c|}{$\mathrm{p}=\mathbf{0 . 0 0 1}$} \\
\hline
\end{tabular}

\section{Results}

Statistically significant changes after the ST in comparison to the basal examination before the ST were found in the following groups and parameters:

The control group showed a slight increase of the IRSA parameter $(4.4 \pm 1.3$ vs. $3.8 \pm 1.4 ; \mathrm{p}=0.006)$ after the spa treatment (Fig. 1).

Several changes were detected after a spa treatment in the group of patients with well compensated
DM II: increased heart rate $(80.9 \pm 11.0$ vs. $74.6 \pm 9.6$; $\mathrm{p}=0.028)$, reduced IC $(1.2 \pm 0.6$ vs. $2.9 \pm 1.4 ; \mathrm{p}=0.027)$ and reduced $\mathrm{IM}(9.9 \pm 7.4$ vs. $18.0 \pm 6.3 ; \mathrm{p}=0.041)$ (Figs 2,3 and 4).

Patients with a poorly compensated DM II showed a decreased IM (10.9 \pm 8.6 vs. $16.9 \pm 5.2 ; \mathrm{p}=0.001)$ and simultaneously also a reduced IRSA (4.1 \pm 3.5 vs. 6.3 $\pm 1.9 ; \mathrm{p}=0.001$ ) (Figs 5 and 6).

There were no significant changes $(p<0.05)$ in other electrocardiographic parameters (Table 1). 


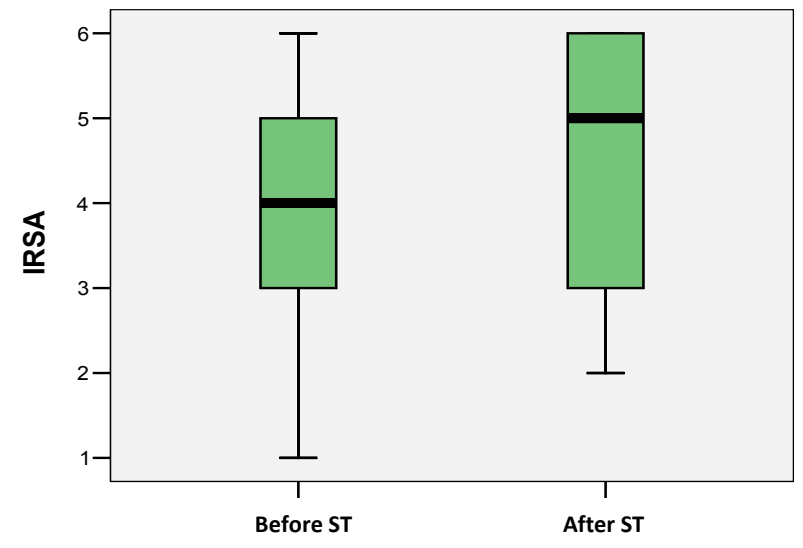

Fig 1. Control group, IRSA (ST - spa treatment).

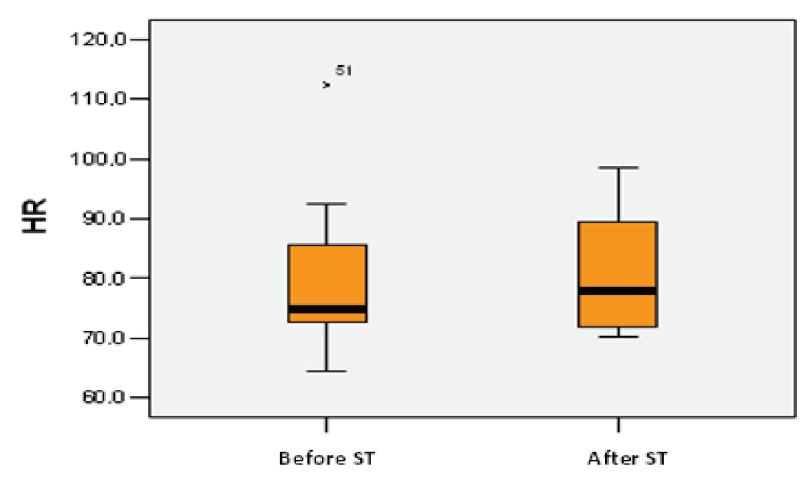

Fig 3. Compensated DM II group, HR (ST - spa treatment).

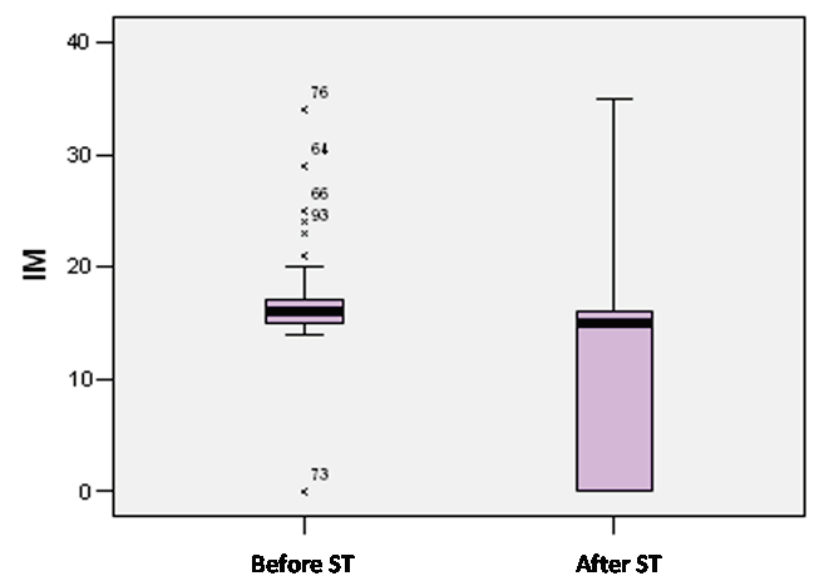

Fig 5. Poorly controlled DM II group, IM. (ST - spa treatment).

\section{Discussion}

The main finding of this study is that the spa treatment succeeded in patients with poorly controlled DMII in reducing the sympathoadrenal system activity (decreased IRSA), in improving electrical stability of the myocardium and in increasing centrally controlled heart rate variability (increased IC) without overloading the

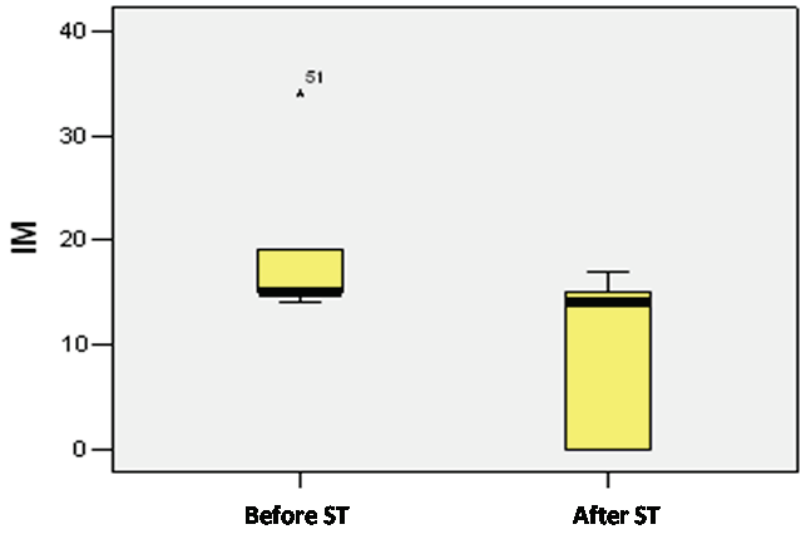

Fig 2. Compensated DM II group, IM (ST - spa treatment).

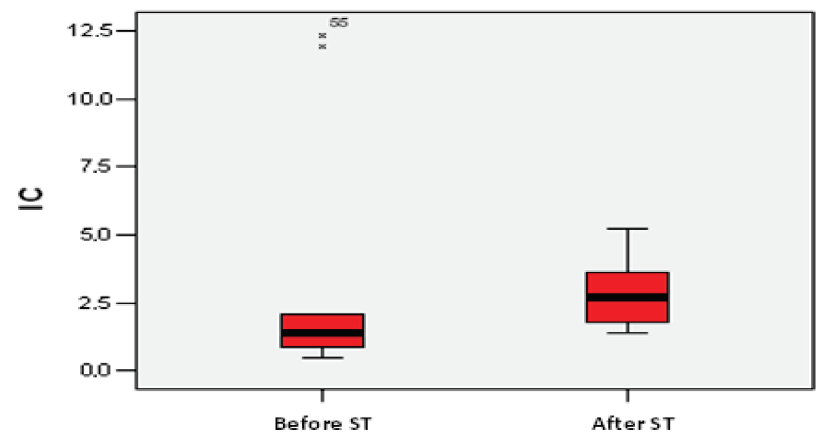

Fig 4. Compensated DM II group, IC. (ST - spa treatment).

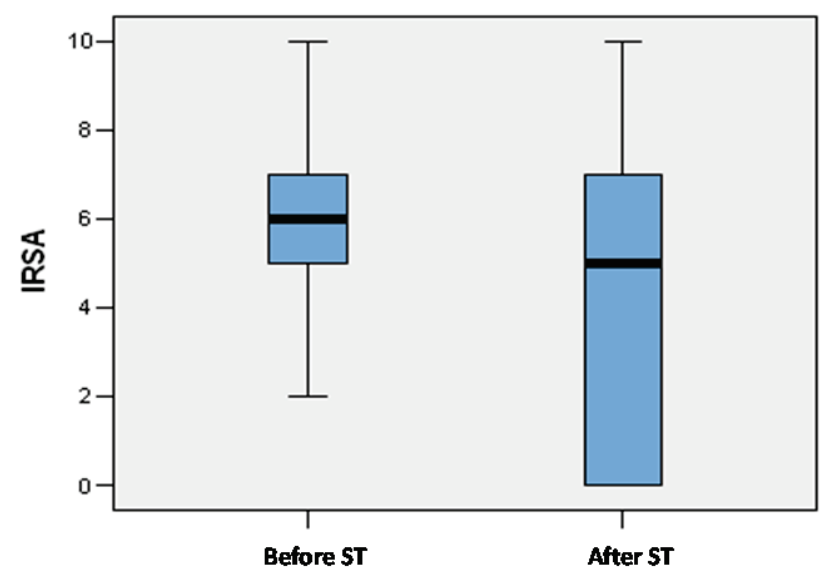

Fig 6. Poorly controlled DM II group, IRSA. (ST - spa treatment).

cardiovascular system (drop of IM).

The metabolic disorder of diabetes leads to damage of peripheral and autonomic nerves. Therefore, it can be suspected that a patient diagnosed with diabetes mellitus type 2 should have at least subclinical disturbances of the autonomic nervous system (Vinik et al. 2003). The autonomic nervous system (ANS) closely integrates vital processes such as heart rate, blood 
pressure and myocardial contractility and as a consequence plays a pivotal role in the regulation of the cardiovascular system (Bissinger et al. 2014). Two important factors of balneotherapy that have an effect on the ANS are $\mathrm{CO}_{2}$ and a regular exercise. Recent study of Bissinger et al. (2014) has proved that improvement of glycemic control leads to improvement of HRV parameters. As no significant change of glycaemia was identified during the ST in our study, a direct effect of these two factors of the ST on the ANS can be supposed.

Carbon dioxide is not only the end product of intracellular metabolism but an active participant in various reactions as well. It affects the biosynthetic processes, redox processes and participates in an energetic exchange of the intracellular metabolism. Sometimes, it behaves as a substrate of reactions in tissues and intracellular fluids, and sometimes as a catalyst of biochemical processes. When applied, it locally decreases the viscosity of blood in small capillaries and flexibility of erythrocytes. This leads to vasodilation which improves blood circulation (Jandova 2009). The result is an increased cardiac output by $30-50 \%$ in spite of a decrease in the heart rate, the heart pressure load is reduced in favor of an economically favorable volume load of the heart what results in a prolongation of a diastole and increased economy of the myocardial function. The changes in HRV indicate that after a complex bath in natural mineral water with carbon dioxide, the tone of ANS is changed towards decreasing the sympathetic tone and the ratio between the sympathetic and parasympathetic is in favor of the parasympathetic (Jandova 2009).

Thanks to a regular exercise, the levels of catecholamine in the plasma and urine decrease and the high setting of the sympathetic tone is reduced. During the balneological procedures, the baroreflex sensitivity is increased. Baroreflex sensitivity is closely dependent on the ANS function in both heart functions and blood vessels management, particularly in controlling of the blood pressure and the heart rate. Regular physical activity leads to a predominance of the parasympathetic regulation (Jandova 2009). It has been shown by analyzing HRV and baroreflex sensitivity that cardiovascular adaptation mechanisms are severely impaired in patients with long-term DM (Lengyel et al. 1998). Our study revealed that both HRV and microwave alternans parameters were found to be significantly improved in DM II patients during balneotherapy. This corresponded inter alia to the findings of positive changes of spectral HRV parameters induced by regular physical activity during balneotherapy what can be supposed to be an evidence of a health improvement (Jandova 2009, Xhyheri et al. 2012).

After the spa treatment of our control group, there was only a slight statistically significant increase in the value of IRSA within one functional status 3-4, suggesting a state of moderate tension of ANS regulatory systems which can be explained as a consequence of an adaptation to the overall load of the spa treatment. This finding can be due to the regular exercise as physical training was proved to decrease cardiac parasympathetic nervous system activity in diabetic patients (Roy et al. 1989). The group of diabetic patients showed a reduction of IM which indicates a reduction of the dispersion deviation and thus an improvement of the electrical stability of the myocardium. The increase in the HRV parameters and IC suggested an increased prevalence of non-respiratory sinus arrhythmia compared to the respiratory ones, and thus an overall increase of the ratio between the central regulation of the autonomic cardiac rhythm and the autonomous regulatory level. The respiratory system can be considered as a part of the response of the autonomic level of regulation of the heart rate. The regulatory control level is characterized by various slow oscillations of the heart rhythm. Its indicator is the non-respiratory sinus arrhythmia. The direct connection between controlling and controlled levels is mediated by nerves (especially sympathetic) and humoral channels. The response has also a nervous and humoral pathway and afferent impulses from baroreceptors of the heart and vessels, from chemoreceptors and large receptor areas of other organs and tissues play a significant role. Local level of control operates under idle conditions in an independent mode which is characterized by a significant respiratory arrhythmia. These respiratory waves are amplified for example during sleep or anesthesia, where the central influence on the autonomic level of control is reduced. Various organism stresses require the intervention of the central level of control and lead to weakening the respiratory sinus arrhythmia components and strengthening the non-respiratory components (Roy et al. 1989). This explains why the observed changes appear as a response to the spa treatment load without overloading the cardiovascular system, yet improving the electrical stability of the myocardium.

Two parameters, IRSA and IM, were decreased when treating poorly controlled DM II patients after the 
spa therapy (ST). This indicates the transition from a functional condition with a considerable tension of the regulatory systems which is connected to an active mobilization of protective mechanisms, including an increased sympathetic activity and pituitary-adrenal system, to a functional condition with only a slight tension of the regulatory systems and, moreover, to a reduction of the dispersion deviation of the myocardium. Cardiac autonomic neuropathy is a severe and relatively early complication of DM II (Vinik et al. 2003, Boulton et al. 2005). In diabetic patients with autonomic neuropathy, a decrease in parasympathetic tone occurs and the predominance of sympathetic activity may predispose these patients to cardiovascular events (Bernardi et al. 1992). For this reason, identification of early and/or asymptomatic autonomic neuropathy may be important for cardiovascular risk stratification in the diabetic population and its medical compensation can positively influence the level of cardiovascular risk (Spallone et al. 1993). Therefore, our results confirm that, due to the ST, there was a decrease in the regulatory systems tension, a shift of the vegetative balance towards the parasympathetic part of the autonomic nervous system, an improvement of the perfusion and the stability of the myocardium, and thereby, an overall subjective improvement of the patients' conditions and compensation of cardiovascular and metabolic risks. Nevertheless, all these findings must be validated in future by bigger number of DM patients.

\section{Conclusion}

The results proved favorable changes in the ANS cardiovascular control of patients with DM II after a spa treatment, especially in terms of reducing the sympathoadrenal system activity (decreased IRSA), improving electrical stability of the myocardium and increasing centrally controlled heart rate variability (increased IC) without overloading the cardiovascular system (drop of IM).

\section{Conflict of Interest}

There is no conflict of interest.

\section{Acknowledgements}

This study was supported by grants PRVOUK-P35/LF1/5 and SVV 260021.

\section{References}

BAEVSKII RM, BARSUKOVA ZV, IOSELIANI KK, SEMENOVA TD: Arterial pressure and autonomic regulation of the heart during simulation of strenuous operator activity. Hum Physiol 9: 301-305, 1983.

BAEVSKY RM, PETROV VM, CORNELISSEN G, HALBERG F, ORTH-GOMER K, AKERSTEDT T, OTSUKA K, BREUS T, SIEGELOVA J, DUSEK J, FISER B: Meta-analyzed heart rate variability, exposure to geomagnetic storms, and the risk of ischemic heart disease. Scr Med (Brno) 70: 201-206, 1997.

BERNARDI L, RICORDI L, LAZZARI P, SOLDA P, CALCIATI A, FERRARI MR, VANDEA I, FINARDI G, FRATINO P: Impaired circadian modulation of sympathovagal activity in diabetes: a possible explanation for altered temporal onset of cardiovascular disease. Circulation 86: 1443-1452, 1992.

BISSINGER A, RUXER J, AHMED RB, LUBINSKI A: Heart rate turbulence in patients with poorly controlled diabetes mellitus type 2. Arch Med Sci 10: 1073-1077, 2014.

BOULTON AJ, VINIK AI, AREZZO JC, BRIL V, FELDMAN EL, FREEMAN R, MALIK RA, MASER RE, SOSENKO JM, ZIEGLER D: Diabetic neuropathies: a statement by the American Diabetes Association. Diabetes Care 28: 956-962, 2005.

HENNERSDORF MG, KELM M, SCHANNWELL CM, RÖSEN P, STRAUER BE: Cardiac complications in diabetes mellitus, Med Klin (Munich) 95: 487-495, 2000.

IVANOV GG, KOVTUN VV, KAGO M, TITOMIR LI: High resolution electrocardiographic abnormalities as possible indexes of electric instability of the myocardium. Can J Cardiol 12: 53-58, 1996.

JANDOVA D: Balneology. (in Czech) Grada Publishing, Prague, 2009.

LENGYEL C, TOROK T, VARKONYI T, KEMPLER P, RUDAS L: Baroreflex sensitivity and heart-rate variability in insulin-dependent diabetics with polyneuropathy. Lancet 351: 1436-1437, 1998. 
PALOVA S, SZABO K, CHARVAT J, SLAVÍCEK J, MEDOVÁ E, MLČEK M, KITTNAR O: ECG body surface mapping changes in type 1 diabetic patients with and without autonomic neuropathy. Physiol Res 59: 203-209, 2010.

ROY TM, PETERSON HR, SNIDER HL, CYRUS J, BROADSTONE VL, FELL RD, ROTHCHILD AH, SAMOLS E, PFEIFER MA: Autonomic influence on cardiovascular performance in diabetic subjects. Am J Med 87: 382-388, 1989.

SPALLONE V, BERNARDI L, RICORDI L, SOLDA P, MAIELLO MR, GAMBARDELLA S, FRATINO P, MENZINGER G: Relationship between the circadian rhythms of blood pressure and sympathovagal balance in diabetic autonomic neuropathy. Diabetes 42: 1745-1752, 1993.

STUCKEY MI, PETRELLA RJ: Heart rate variability in type 2 diabetes mellitus. Crit Rev Biomed Eng 41: 137-147, 2013.

VINIK AI, MASER RE, MITCHELL BD, FREEMAN R: Diabetic autonomic neuropathy. Diabetes Care 26: 1553$1579,2003$.

XHYHERI B, MANFRINI O, MAZZOLINI M, PIZZI C, BUGIARDINI R: Heart rate variability today. Prog Cardiovasc Dis 55: 321-331, 2012.

ZDARSKA D, PELISKOVA P, CHARVAT J, SLAVICEK J, MLCEK M, MEDOVA E, KITTNAR O: ECG body surface mapping (BSM) in type 1 diabetic patients. Physiol Res 56: 403-410, 2007.

ZIEGLER D: Diabetic cardiovascular autonomic neuropathy: prognosis, diagnosis and treatment. Diabetes Metab Rev 10: 339-383, 1994. 